\title{
Identification and Diagnosis of Fault in Consistent Mixed Tank Reactor using Neural System
}

\author{
R. Gowthami, I. Aravindaguru, V.M.Hari
}

\begin{abstract}
The two horrible conditions ahead of time than the organizers worldwide are to diminish the stack at the conventional fills and to reduce the continually developing basic spoiling. This test is proposed to discover probably the execution of the DI diesel motor at various loads when fuelled with mixes of palm methyl esters and diesel. The primers have been pushed on a completely utilized diesel motor without changes. Every one of the appraisals were consistent usa of america and outfitted toward dependable pace. The impact of moving weight develop to be assessed the volume that brake warm temperature ability, mass flow rate, brake one of a kind gas use and fumes gas temperature. Exploratory impacts show that at complete weight conditions, the B-20, B-40and B-60 mixes bring 33.23\%, 32.81\%, $32.39 \%$ and 31.ninety seven\% higher brake heat usefulness than sole diesel freely. It wound up confirmed that the brake warmth ability of palm biodiesel is higher than that of diesel, and it is a delayed consequence of the oxygenated atom of biodiesel which acknowledges total ingesting of the biodiesel fuel. In addition the mass development rate of biodiesel is evidently superior to anything that of diesel fuel; it is through method for exact capacity of the calorific estimation of biodiesel is a ton parcels less appeared in one another way as far as diesel gas. At the reason for results obtained from this test utilizing palm biodiesel as a fuel is proposed for the utilized as a piece of a diesel motor with diesel mixes.
\end{abstract}

Keywords: biodiesel, esterification, pyrolysis, emulsification, blends.

\section{INTRODUCTION}

The fundamental data about Consistent Mixed Tank Reactor procedure is that, it is very nonlinear procedure, irreversible first request framework and furthermore it is exothermic strategy. Consistent Mixed Tank Reactor tank will work at unfaltering state condition and furthermore utilized for well blending reason. In this way, once Consistent Mixed Tank Reactor tank begins, the reactants are included into the procedure tank, the stirrer will mix the reactants to give the ideal item. A Consistent Mixed Tank Reactor which is an ideal model which can be utilized for blending reason in pharmaceutical enterprises. When the Consistent Mixed Tank Reactor tank begins, the end result items are included into the procedure tank, the stirrer is utilized to blend the reactants to give the ideal last item. A Consistent Mixed Tank Reactor tank is an ideal model is utilized to comprehend and gauge the individual procedure factors by using nonstop disturbance of reactor. In this

Revised Manuscript Received on August 14, 2019.

R. Gowthami, Department of Electronics and Instrumentation Engineering, M. Kumarasamy College of Engineering, Karur, Tamilnadu, India.

I. Aravindaguru, Test Analyst, IBM, Bangalore., Karnataka, India.

V.M.Hari, Test Analyst, IBM, Bangalore, Karnataka, India manner, the ideal yield can be accomplished. For low and for high scale creation the Consistent Mixed Tank Reactor tank tumult procedure will be used. The Consistent Mixed Tank Reactor tank utilizes three control circles which is utilized to direct the output temperature and straight stream rate of the element of the input that we given inside the tank While blending, the warmth will be produced during the procedure inside the Consistent Mixed Tank Reactor tank. Along these lines, the coolant will be made to course through the outlet coat around the reactor. Indeed, subsequent to avoiding potential risk steps the issue will happen in the Consistent Mixed Tank Reactor tank which may prompts off base outcome. The shortcoming is said to be a startling change in the framework which may prompt a deficiency in the physical segments, for example, sensors and in actuator. Along these lines, the Neural System Prescient Controller can be utilized to distinguish and conclusion different sorts of flaws which may happen in the Consistent Mixed Tank Reactor tank.

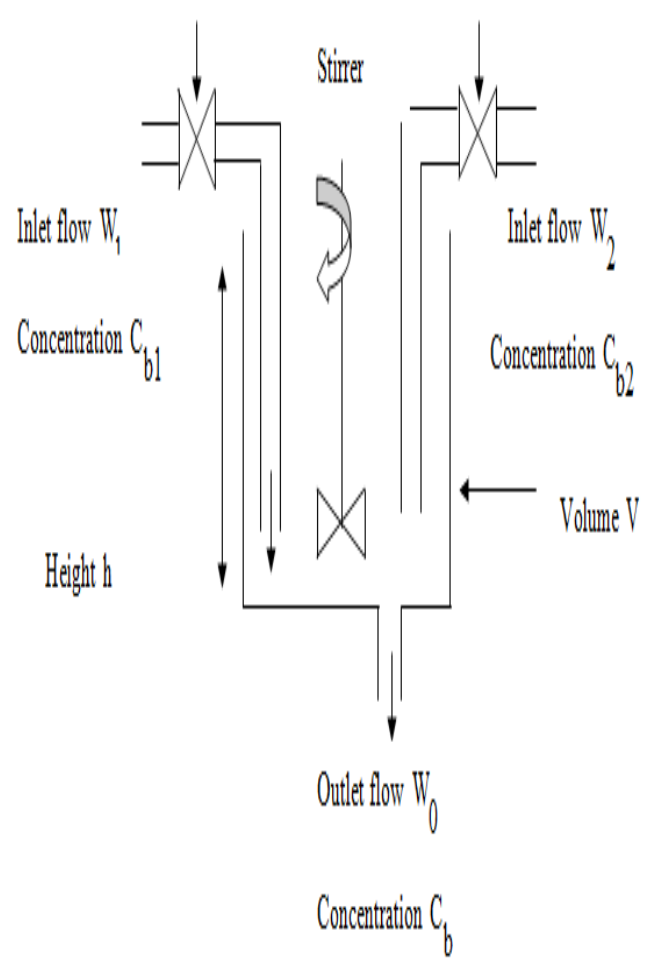

Fig.1: Basic CSTR tank

Published By: Blue Eyes Intelligence Engineering 


\section{MODELING OF CONSISTENT MIXED TANK REACTOR TANK}

The scientific model for the procedure is give the conduct of the procedure. From the numerical model of a specific procedure the attributes of different parameters can be comprehend in an unmistakable way. When the reactants are included into the tank which will mix the reactants well and it will create wanted precise item as yield. During this procedure, the warmth will be created which can be utilized to continue the procedure. In the event that the warmth delivered in the tank which builds implies the coolant water will be permitted to stream in the external coolant coat which is put around the tank. So some measure of hotness inside the tank can be decreased. In this paper the Consistent Mixed Tank Reactor tank taken will have the two sources of info reactants utilized are W1 which go about as information stream rate of concentrated feed and W2 will go about as the channel stream rate of weakened feed. Along these lines, both $\mathrm{W} 1$ and $\mathrm{W} 2$ are reactants to the CSTR tank.

By utilizing the mass equalization condition

The flow of progress of mass in the Consistent Mixed Tank Reactor system=The flow of mass stream inlet - flow of mass stream outlet

Where W0 will act as outlet flow rate

$\mathrm{dh} / \mathrm{dt}=\mathrm{M} 1+\mathrm{M} 2-\mathrm{W} 0$

$\mathrm{W} 0=\mathrm{CV}^{*}(\mathrm{~h})^{\wedge} 1 / 2$

$\mathrm{dh} / \mathrm{dt}=\mathrm{M} 1+\mathrm{M} 2-\mathrm{CV}^{*}(\mathrm{~h})^{\wedge} 1 / 2$

When $\mathrm{CV}=0.2$

By substituting $\mathrm{CV}=0.2$ in the equation 4 . We get,

$\mathrm{dh} / \mathrm{dt}=\mathrm{M} 1+\mathrm{M} 2-(0.2)^{*}(\mathrm{~h})^{\wedge} 1 / 2$

Where

$$
\mathrm{h}=\text { Liquid level }(\mathrm{cm})
$$

M1=Inlet flow rate of the concentrated feed $\left(\mathrm{cm}^{3} / \mathrm{s}\right)$

$\mathrm{M} 2=$ Inlet flow rate of the diluted feed $\left(\mathrm{cm}^{3} / \mathrm{s}\right)$

$\mathrm{W}_{0}=$ Outlet flow rate $\left(\mathrm{cm}^{3} / \mathrm{s}\right)$

Along these lines of the centralization in the constant mixed tank inlet is given in the accompanying condition

\section{FAULT DIAGNOSIS TECHNIQUES}

The flight of a watched estimation of a variable from the genuine worth is by and large alluded as issue. Fig.2 shows the plant finding by utilizing the sensor. Here the sensor worth made to contrasted and the set point which is predefined in the spot of comparator. In the event that any will prompts give sign to the actuator by the criticism controller. The acquired actuator sign will be given to the deficiency analysis framework. In the event that any peril or flaw happen in the actuator of the analysis framework. The finding framework will deliver alert alarm. This flaw recognition and finding framework likewise have the ability of giving data about the beginning planning, area and seriousness of deficiency in the plant. Those extra highlights which is available in issue finding framework are called as supervision framework. The data got from the flaw finding framework can be utilized for settling on choice for shortcoming happens in the sensor that sign from the sensor

diminishing issue in earlier by rolling out certain improvements to the info and yield of the procedure to get wanted item with no debasement in the nature of the item.

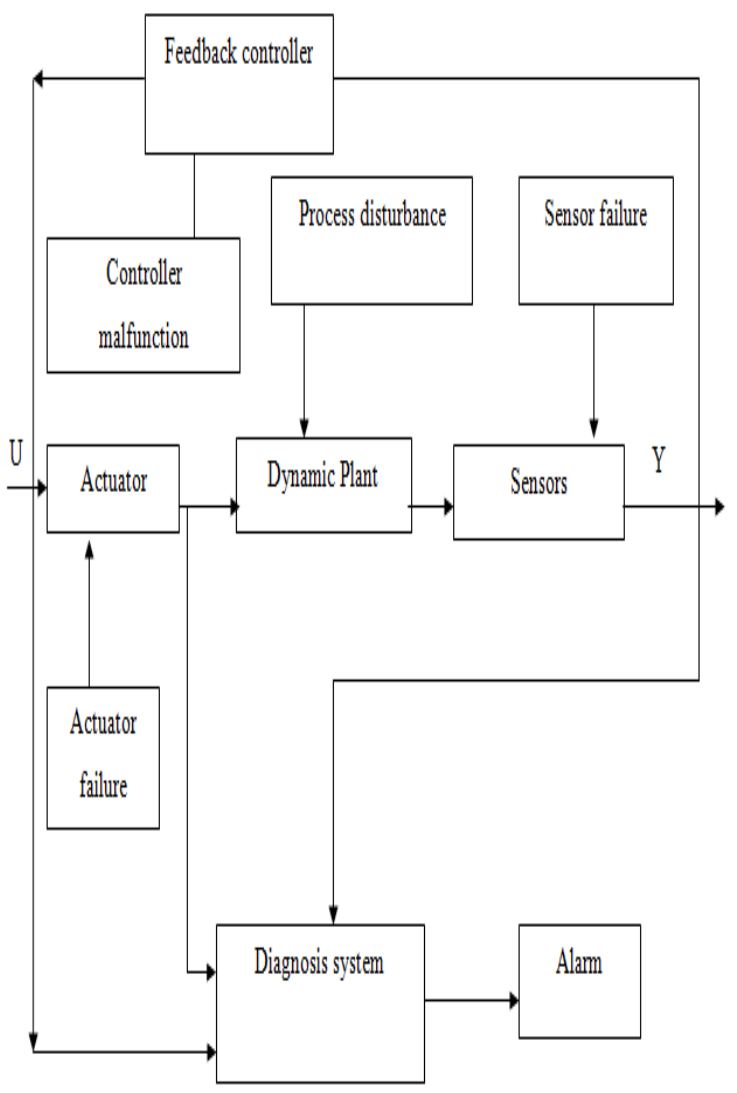

Fig.2 Fault Diagnosis general block diagram

\section{NEURAL SYSTEM PRESCIENT CONTROLLER}

In Consistent Mixed Tank Reactor because of changes in the temperature of the tank can be limited by modifying the coolant stream rate which streams in the external coolant coat. In MATLAB, the Neural System Prescient Controller square is accessible. In that obstruct, the Consistent Mixed Tank Reactor plant model will be stacked. The yield signal from the plant model is given again as sign to the NN plant model. The plant model which additionally use the arbitrary reference signal. By utilizing this reference signal, the square can contrast the plant yield signal and the predefined reference signal so that if any deviation, happen implies the controller will make essential strides. In the wake of associating the plant model with the neural system model, it will give reaction. From the reaction, the attributes of the centralization of the item in the Consistent Mixed Tank Reactor tank can be gotten. By utilizing this Neural System Prescient Controller strategies, the future plant outlet can likewise be anticipated. In the Neural System Prescient Controller the preparation information's can be acquired in a manner like the sequential arbitrary reference step inputs. In the wake of preparing every one of the information's which

Published By: 
is created in the process is helpful for acquiring the servo reaction of the grouping of Consistent Mixed Tank Reactor tankitem.

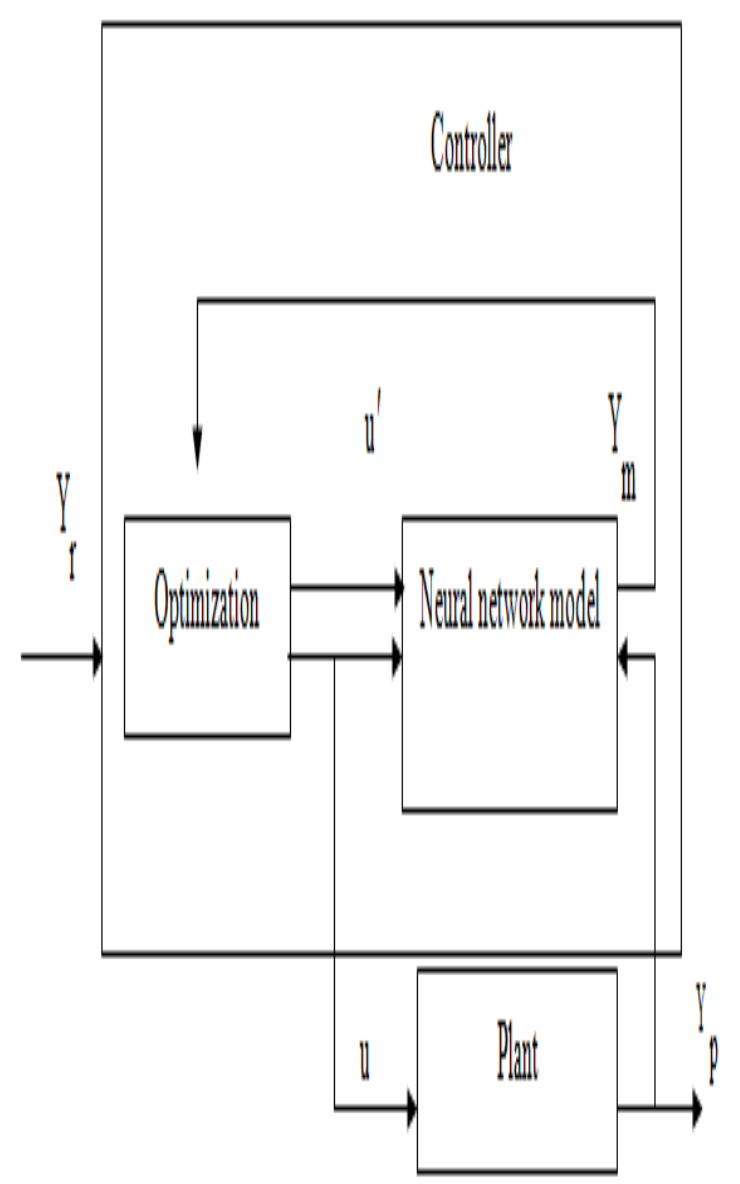

Fig.3: Neural Network model

\section{NEURAL SYSTEM PRESCIENT CONTROLLER \& RESULTS}

The artificial networks is one of the approach for MPC. In general, the MPC uses the neural system based on artificial which is used as the plan system model is used to get the desired prediction outlet. Most commonly utilised technique is the MPC technique. The main reason for using MPC algorithm approach is to utilise system model to understand in prior about the forthcoming control action for production for the process output. Neural System Prescient Controller is mainly based on recent control horizon structure. The mathematical optimization is useful to identify the effect of future control signal.

The skyline over the following of mistake and it likewise helpful to assess the augmentations in the control. The variable for the dubious control system signal, is the required output and is the $\mathrm{NN}$ type response of this framework. This worth is utilized to decide the exhibition list by the expansion of the square of control increases. In the wake of figuring the ideal control succession by actualizing it in the beginning control activity. At that point the skyline will be moved to the forward movement by one inspecting time moment and the data is enhanced. Again began with new subtleties and the procedure will be rehashed. This is like the adjustment in the control. The MPC depends on the neural system plant model and it likewise utilizes improvement framework square. By utilizing MATLAB programming, the Neural System Prescient Controller unit which will give the attributes of the parameters present in the Consistent Mixed Tank Reactor tank. Fig. 4 gives the Neural System Prescient Controller square which incorporates plant framework ID for finding the consistency of definite item inside the Consistent Mixed Tank Reactor tank. Here, the Neural System Prescient Controller square is again connected to the Consistent Mixed Tank Reactor model which likewise incorporates the scientific demonstrating for the Consistent Mixed Tank Reactor tank. The information sign, for example, irregular reference sign is given to the Neural System Prescient Controller. The Neural System Prescient Controller is the graphical client based interfacing instrument. By utilizing Neural System Prescient Controller obstruct, the future plant yields can be anticipated utilizing plant model.

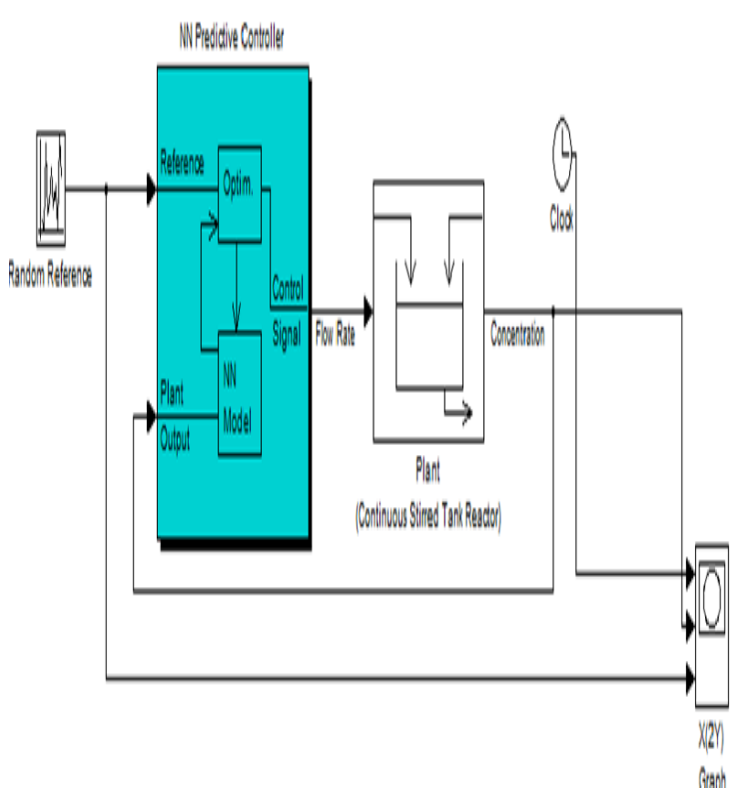

Fig.4: Basic Neural System

\section{Prescient Controller block}

The NN plant model, comprise of one concealed layer. By giving arrangement of reference sign of the plant info and yield information, the information's can be prepared by using trainlm which is appeared in the fig.5. In fig.6 the approval information of Neural System Prescient Controller is appeared. The servo reaction of Consistent Mixed Tank Reactor parameters is appeared in fig.7. In light of the ideal grouping of Consistent Mixed Tank Reactor yield item, the information's will be prepared utilizing trainlm for Neural System Prescient Controller which is appeared in fig. 8 and fig.9. Fig.10 demonstrates the servo reaction of defective focus information of Consistent Mixed Tank Reactor tank. 

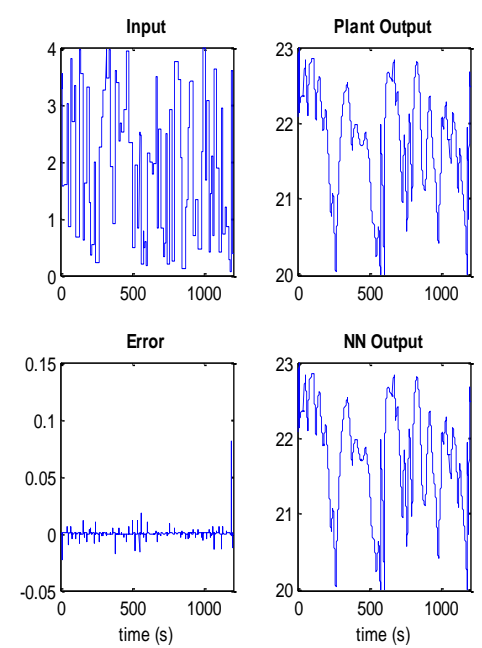

Fig.5: NNPC training data
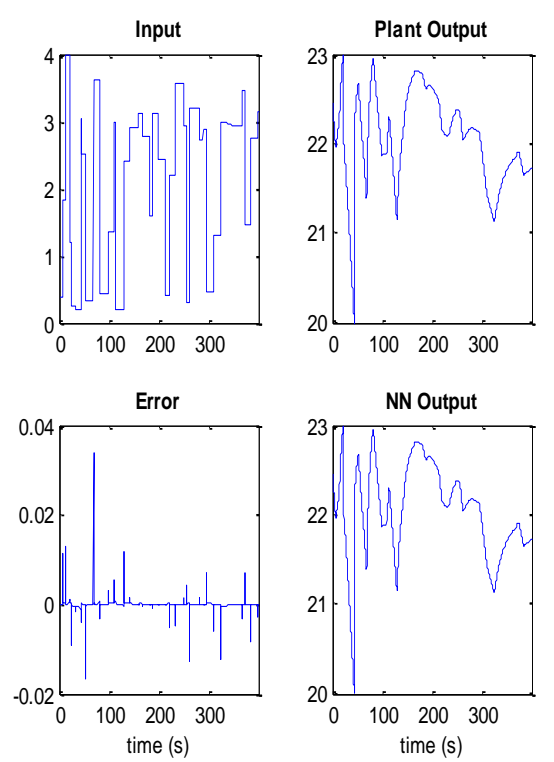

Fig.6 NNPC validation data

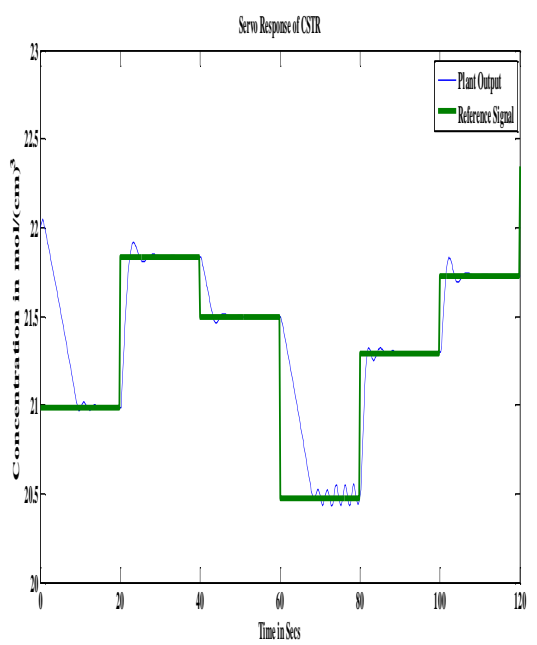

Fig.7: Without fault Servo response of Consistent Mixed Tank Reactor
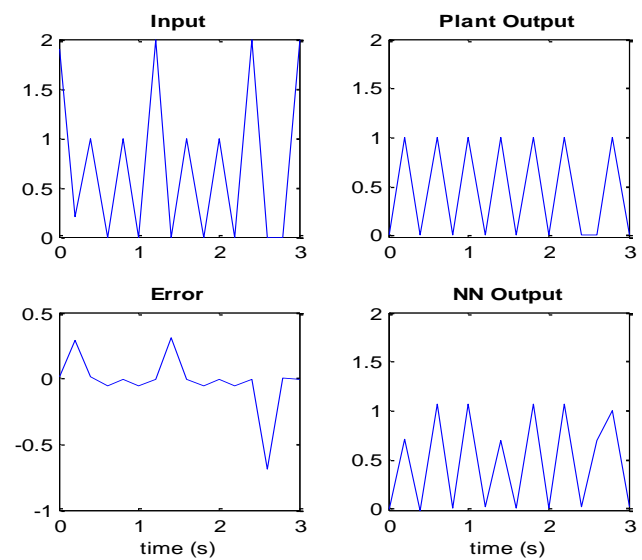

Fig.8: In Neural System Prescient Controller training data
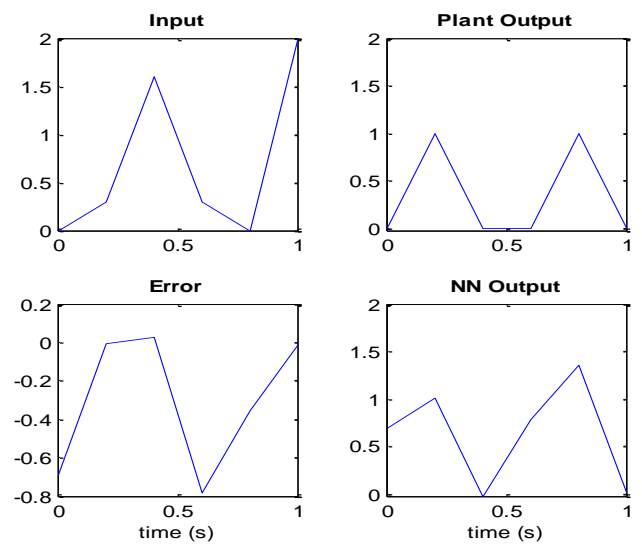

Fig.9: In Neural System Prescient Controller validation data

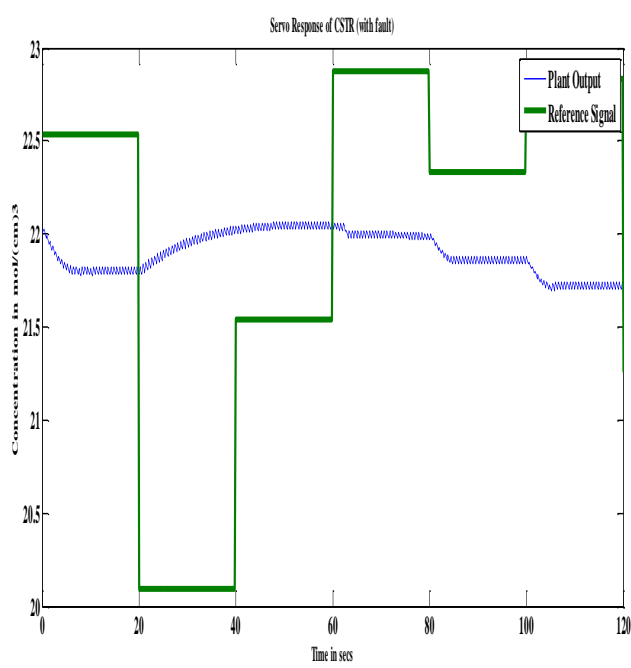

Fig.10: Faulty Servo response for data of Consistent Mixed Tank Reactor tank parameters 


\section{CONCLUSION}

In this way the Consistent Mixed Tank Reactor is a helpful application that is all around usually utilized in different applications. The Consistent Mixed Tank Reactor parameters are adequately displayed utilizing mass parity condition which give a reasonable comprehension of reaction and attributes of every parameter engaged with the Consistent Mixed Tank Reactor process. Different flaws were distinguished and the rate were evaluated by utilizing Neural System Prescient Controller. This deficiency ID and estimation can be additionally broadened utilizing ANFIS system moreover.

\section{REFERENCES}

1. Dong-Juan Li (2014), 'Neural network control for a class of continuous stirred tank reactor process with dead-zone input', Elsevier Journal of Neurocomputing, Vol.131, pp.453-459.

2. Kanse Nitin G., Dhanke P.B. and Thombare Abhijit (2012), 'Modeling and Simulation Study for Complex Reaction by Using Polymath', Research Journal of Chemical Sciences, Vol.2, No.4, pp.79-85.

3. Mehdi Namdari, Hooshang Jazayeri-Rad, Nader Nabhani (2013), 'Comparing the Performance of two Neural Network Methods in a Process Fault Diagnosis System', Journal of Basic and Applied Scientific Research, Vol.3, No.2, pp.942-947

4. Ribhan Zafira Abdul Rahman, Azura Che Soh, Noor Fadzlina binti Muhammad (2010), 'Fault Detection and Diagnosis for Continuous Stirred Tank Reactor Using Neural Network', Kathmandu University Journal of Science, Engineering and Technology, Vol.5, No.2, pp. 66-74.

5. Venkat Venkatasubramanian, Raghunathan Rengaswamy, Kewen Yin, Surya N. Kavuri (2003), 'A review of process fault detection and diagnosis Part I:Quantitative model-based methods', Elsevier Journal of Computers and Chemical Engineering, Vol.27, pp.293311.

6. Venkat Venkatasubramanian, Raghunathan Rengaswamy, Surya N. Kavuri, Kewen Yin (2003), 'A review of process fault detection and diagnosis Part III: Process history based methods', Elsevier Journal of Computers and Chemical Engineering, Vol.27, pp.327346.

7. Vishal Vishnoi, Subhransu Padhee, Gagandeep Kaur (2012), 'Controller Performance Evaluation for Concentration of Isothermal Continuous Stirred Tank Reactor', International Journal of Scientific and Research Publication, Vol.2, No.6, pp.2250-3153.

8. Yunosuke Maki and Kenneth A. Loparo (1997), 'A Neural-Network Approach to Fault Detection and Diagnosis in Industrial Processes', IEEE Transactions on Control Systems Technology, Vol.5, No.6, pp.529-541.

9. Vishal Vishnoi, Subhransu Padhee, Gagandeep Kaur (2012), 'Controller Performance Evaluation for Concentration of Isothermal Continuous Stirred Tank Reactor', International Journal of Scientific and Research Publication, Vol.2, No.6, pp.2250-3153.

10. Monisa, S., Vijayachitra, S., 2015, "Real Time Performance Analysis and Fault Diagnosis in Heat Exchanger", International Journal of Chemtech Research, Vol.8, No.12, pp.418-427

11. Sivashankari V, 2015, 'Damping of power Oscillation Using UPFC with Implementation of Neuro- Fuzzy', International Open Journal of Educational Research, Vol. 1, No. 3, pp. 108-112.
12. P. Yuvarani, 2012, 'Image denoising and enhancement for lung cancer detection using soft computing technique,' IET Chennai 3rd International on Sustainable Energy and Intelligent Systems (SEISCON 2012), Tiruchengode, pp. 1-4. doi: 10.1049/cp.2012.2179. 